\title{
Physical Properties of Mixtures of Rice Starch and Tapioca Starch Prepared by Extrusion Cooking Technology
}

\author{
Wen Xia ${ }^{1, a}$, Ji-hua $\mathrm{Li}^{{ }^{* 1,} \text { b }}$, Fei Wang ${ }^{1, \mathrm{c}}$, Xiao-yi Wei, ${ }^{1, \mathrm{~d}}$, Tiao-kun $\mathrm{Fu}^{1, \mathrm{e}}$ and \\ Zhen-wen Zhang ${ }^{2, f}$ \\ ${ }^{1}$ Agricultural Product Processing Research Institute at Chinese Academy of Tropical Agricultural \\ Sciences, Zhanjiang 524001, China. \\ ${ }^{2}$ Tropical Crops Genetic Resources Institute Chinese Academy of Tropical Agricultural Sciences \\ Hainan Province, China \\ axiawensg@163.com, bfoodpaper@126.com, '185541703@qq.com, dweixywei@163.com, \\ e298108151@qq.com, 'scuta96@163.com
}

\begin{abstract}
Keywords: Extrusion; Tapioca starch; Physical properties; rice starch
Abstract. The purpose of this study is to investigate the physical properties of mixtures of rice starch and tapioca starch (TS) prepared by extrusion cooking technology. The microstructure analysis suggested that samples presented a looser and more porosity structure as TS content increased. The $\mathrm{X}$-ray diffraction patterns of samples could be classified as typical V-type structure, while the crystallinity decreased with increasing TS content. Rapid visco analysis indicated that extruded samples showed a rapid increase in viscosity during the initial heating process and exhibited a lower tendency towards retrogradation on cooling process compared to control. These results provided the basic information on the physical properties of mixtures of rice starch and tapioca starch prepared by extrusion cooking technology.
\end{abstract}

\section{Introduction}

Tapioca starch (TS) is widely produced in Latin America and also one of the most important industrial crops in Thailand. It shows lower pasting temperature, higher viscosity [1], higher amylopectin content and relatively lower price when compared to other starch in the world market [2], which makes it suitable for food industries. CS has been widely used in many places, such as thickening or gelling agent in food engineering [3]. But TS is still used to a lesser extent when compared to the other starches, like corn starch in the food industry [4].

Extrusion is an important technique which was used worldwide for the production of ready-to-eat cereal foods, modified starch and porridge [5]. Extrusion is a thermal processing with a combination of high heat, high pressure and shear forces [6], resulting numbers of chemical changes that occur, including gelatinization of starch, the generation of flavors and cross-linking of proteins . However, only a few reports study the mixtures of rice starch and tapioca starch prepared by extrusion cooking technology(ECT). The objective of this research was to study the mixtures of rice starch and tapioca starch prepared by ECT, and its physical properties were investigated.

\section{Experimental}

Materials. The purity degree of tapioca starch (Thai World Import and Export, Bangkok, Thailand) and rice starch were 99.7\% (dry weight basis) and 99.6\%, and they were purchased from a food factory in Thailand and China, respectively. All chemicals used were of analytical grade.

Preparation of blended samples and treatment by extrusion cooking technology. The experiment was carried out according to the method of Zhang et al., (2014) [7].

SEM analysis. Microstructural images were obtained from scanning electron microscope (S-4800, Hitachi Limited, Tokyo, Japan) at 1000 magnification and an accelerating voltage of $3.0 \mathrm{kV}$

XRD analysis. X-ray diffraction pattern was measured using a Siemens D5000 X-ray diffraction instrument (Rigaku Corporation, Japan). X-ray diffractograms were obtained under conditions of 36 $\mathrm{KV}, 30 \mathrm{~mA}$, with the scanning angle $2 \mathrm{q}$ set from $5^{\circ}$ to $40^{\circ}$ at a scanning rate of $0.6 \% \mathrm{~min}$. 
RVA analysis. Samples (2.8 g; 12\% moisture) were determined with a rapid visco analyser (Newport Scientific, Warriewood, NSW, Australia) and slurried with distilled water (25 mL). The parameters setting were performed according to the method of Xia et al. (2014) [8].

\section{Results and disscussion}

SEM analysis. Samples were analyzed through SEM and the sectional micrographs are given in Fig.1, it was found that control sample (Fig.1A) showed a compact and intact structure. However, samples after extrusion presented a looser structure with fewer pores being compared with control. The result may be attribute to that extrusion is a thermal processing with a combination of high heat, high pressure and shear forces, which leads to the gelatinization and degradation of starch [9], and then results in a loose texture.

As the TS content increased, the appearance obtained in samples were looser with more pores (Fig.1 B, C, D and E). It may be ascribed to TS having a good water-binding capacity [10], which retained more water during extrusion. When samples with similar initial moisture (30\%) was dried to the same final moisture (12\%), more pores were observed with increasing TS content after syneresis in drying process[11].

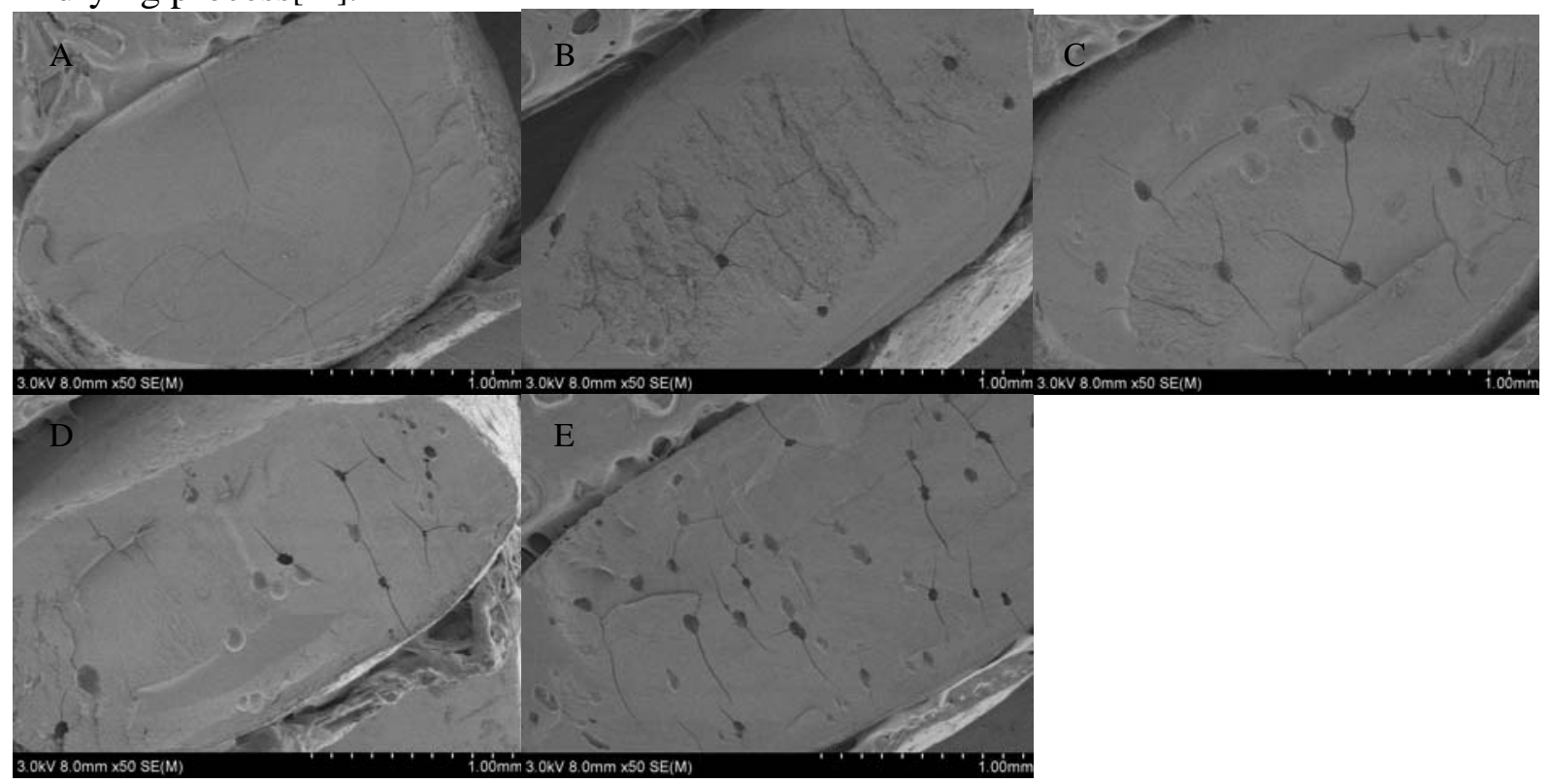

Fig.1 SEM images of samples: A (0\% TS), B (10\% TS), C (20\% TS), D (30\% TS) and E ( $40 \%$ TS)

XRD analysis. The XRD diffractograms of samples are shown in Fig.2 The samples presented typical V-type with a small peak at an angle value of $20^{\circ}$. This peak was identifiable with the main formation of amylose-lipid complexand the intensity of this diffraction peaks decreased with increasing amount of TS. It might be due to the decrease of amylose in extruded samples, causing a reduction in forming the complex of amylose-lipid. Similar results were also obtained by Bhatnagar and Hanna (1994) [12] that the peak heights for V-type patterns decreased with decreasing amylose content. The relative crystallinity decreased from $30.2 \%$ for A to $22.3 \%$ for $\mathrm{B}, 16.6 \%$ for $\mathrm{C}, 12.7 \%$ for $\mathrm{D}$, and $9.11 \%$ for E. It indicated that extruded samples exhibited a lower relative crystallinity in comparison with control. It may be due to the decrease of lipid and protein with the increase of TS in mixtures, which may be easier to gelatinize and degrade during extrusion[13], resulting in a decrease of relative crystallinity. 


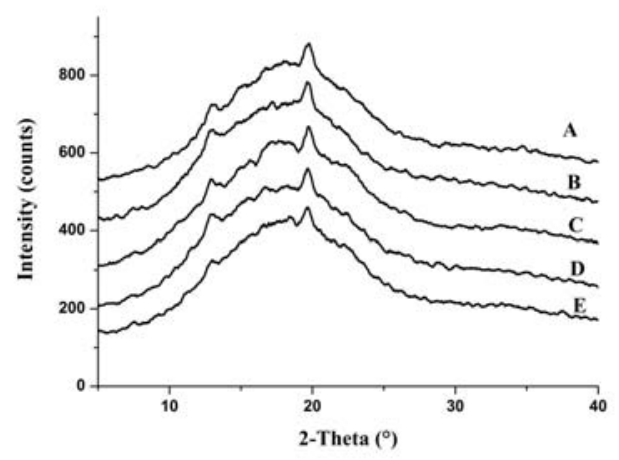

Fig.2 X-ray diffraction patterns of five samples: A (0\%), B (10\% TS), C(20\% TS), D (30\% TS), E (40\% TS)

RVA analysis. RVA is an effective method to demonstrate the pasting properties of starch during heating and cooling process of starch-based products. The pasting patterns of all samples are given in Fig.3. During the initial heating process, initial paste viscosities of samples after extrusion rapidly increased and reached peak earlier than control sample, indicating that samples after extrusion was more accessible for water. It may be due to the loose texture of samples after extrusion which was confirmed from the result of SEM, allowing water molecules to interact with samples after extrusion more easily and swell well. However, the curve of samples after extrusion were different from control. This may be that starches after extrusion were partly gelatinized and did not contain intact starch granules. Thus, the RVA curve of samples after extrusion appeared to be irregular .

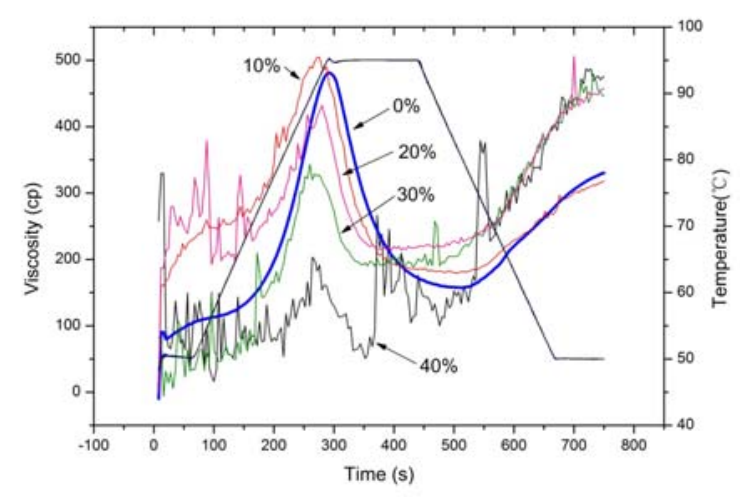

Fig.3 The RVA patterns of samples

\section{Conclusions}

RVA analysis suggested that extruded samples showed a rapid increase in viscosity during the initial heating process and irregular curve compared to control. The microstructure of extruded samples presented a looser and more porosity structure as TS content increased, which indicated that it would be easier cooked and fortified with functional ingredients. The data of this work may supply knowledge of TS on the partial substitution of rice starch in rice or rice flour used in food industry.

\section{Acknowledgements}

The authors gratefully acknowledged the Financial Support by the Fundamental Scientific Research Funds for Chinese Academy of Tropical Agricultural Sciences (Project no.1630032015004) and the Fundamental Scientific Research Funds for Chinese Academy of Tropical Agricultural Sciences (No.1630062013012). 


\section{References}

[1] Knight, J.W. Cassava Processing and Storage. International Development Research Centre (pp. 76). Canada: IDRC,1974.

[2] Rapaille, A., Vanhemelrijck, J. Modified starch. In A. Imeson (Ed.), Thickening and gelling agents for food (pp. 199-229). London: Blackie Academic and Professional,1997.

[3] Moore, C.O., TuschhoV, J.V. Hastings, C.W., Schanefelt, R.V., Applications of starches in foods. In R. L. Whistler, J. N. Bemiller, \& E.F. Paschall, Eds., Starch: Chemistry and technology, Academic Press, Orlando, 1984, 575-591.

[4] FAO. Proceedings of the validation forum on the global cassava development strategy, Global cassava market study business opportunities for the use of cassava 6, Rome,2004.

[5] Gujral, H. S., Singh, N., Singh, B. Extrusion behaviour of grits from flint and sweet corn. Food Chemistry, 2001, 74, 303-308.

[6] Riha, W. E., Hwang, C. F., Karwe, M. V., Hartman, T. G., Ho, C. T. Effect of cysteine addition on the volatiles of extruded wheat flour. Journal of Agricultural and Food Chemistry, 1996, 44, 1847-1850.

[7] Zhang, Y.J., Liu, W., Liu, C.M., Luo, S.J., Li, T., Liu, Y.F. Retrogradation behaviour of high-amylose rice starch prepared by improved extrusion cooking technology. Food Chemistry 2014, 158, 255-261.

[8] Xia, W., Fu, G. M., Liu, C. M., Zhong, Y. J., Zhong, J. Z., Luo, S J., et al. Effects of cellulose, lignin and hemicellulose on the retrogradation of rice starch. Food Science and Technology Research, 2014, 20, 375-383.

[9] Singh, B., Sekhon, K. S., Singh, N. Effects of moisture, temperature and level of pea grits on extrusion behaviour and product characteristics of rice. Food Chemistry, 2007,100, 198-202.

[10] Charoenrein, S., Preechathammawong, N. Effect of waxy rice flour and cassava starch on freeze-thaw stability of rice starch gels. Carbohydrate Polymers, 2012, 90, 1032- 1037.

[11] Charoenrein, S., Preechathammawong, N. Undercooling associated with slow freezing and its influence on the microstructure and properties of rice starch gels. Journal of Food Engineering, 2010, 100, 310-314.

[12] Bhatnagar, S., Hanna, M.A. Amylose-Lipid Complex Formation during Single-Screw Extrusion of Various Corn Starches. Cereal Chemistry, 1994, 71, 582-58.

[13] Gunaratne, A., Bentota, A., Cai, Y.Z., Collado, L. Corke, H. Functional, digestibility, and antioxidant properties of brown and polished rice flour from traditional and new-improved varieties grown in Sri Lanka. Starch/Starke, 2011, 63, 485-492. 